\title{
The Research on Merging Modes of Toll Square Based on Cellular Automata Algorithm
}

\author{
Dongxu Chen
}

North China Electric Power University, Baoding 071000, China

e-mail:chendongxu0000@126.com

Keywords: Toll Square, Accident Rate, Cellular Automata, Merging Mode

Summary: In this paper, we firstly consider the impact of the accident on the problem of toll square traffic and establish a prediction model of traffic conflict to get an accident rate. Then, these parameters are applied to cellular automata model based on the cellular theory, and MATLAB is used to simulate the cell model. According to the result of simulation, we are to analyze and evaluate the toll square, and put forward a kind of ideal merging mode.

\section{Introduce}

Toll square is a vital part of the high way. Its operating efficiency directly affects the level of highway transportation. Low operating efficiency could cause congestion, and even arise traffic accidents. Increasing the toll speed of toll square on the premise of ensuring security and economy is very advantageous for drivers and toll square managers. Therefore, it is necessary to explore how to make rational construction to improve operating efficiency of the toll square. In this paper, we will study the best merging mode of the toll square.

\section{The Model of Traffic Conflict Prediction}

We build a model of traffic conflict prediction based on different factors. We use the running speed, traffic, traffic composition as a variable for building the model. Then, these independent variables were analyzed for correlation.

According to the independent variable correlation analysis results, based on the statistical analysis of traffic flow statistics, predictive model of varying lane conflict for highway toll station is established based on traffic flow parameters. The model can be expressed as follows:[1][2]

$$
G=\exp \left(0.864+0.00245 x_{q}+2.442 x_{b}+0.889 x_{r}\right)
$$

Where: $G$ is occurring numbers predicted value of lane changing conflict.

$x_{q}$ is Equivalent traffic volume.

$x_{b}$ is Proportion of large vehicles.

$x_{r}$ is the proportion of waiting for vehicles to change lanes.

According to the same theory, predictive model of rear - end conflict for highway toll station is established based on traffic flow parameters. The model can be expressed as follows:

$$
F=\exp \left(0.759+0.00262 x_{q}+2.445 x_{b}\right)
$$

Where: $F$ is occurring numbers predicted value of rear - end conflict.

$x_{q}$ is Equivalent traffic volume.

$x_{b}$ is Proportion of large vehicles.

As can be seen from the above description, predictive model of traffic conflict for highway toll station is as follows:

$$
N=\exp \left(0.864+0.00245 x_{q}+2.442 x_{b}+0.889 x_{f}\right)+\exp \left(0.759+0.00262 x_{q}+2.445 x_{b}\right)(3)
$$

Where: $N$ is the predictive number of traffic conflicts occurred in Toll Plaza

Based on the above analysis, we define the accident rate $\beta$, the expression of which is as follows:

$$
\beta=\frac{N}{\varepsilon}
$$


Where: $\varepsilon$ is throughout.

\section{The Model of Cellular Automata}

The Hypothesis of Model. Toll plaza has three types of cell components: occupied cells, vacant cells and forbidden cells. These three kinds of cellular, together with cellular boundary itself can build out a complete simulation of the toll station.

Assume that all vehicles have the same size. Today, large trucks often choose the night traffic for driving safety and relief tolls booths. The main purpose of the private car in small size is travel, they often choose the daytime travel considering the comfort of travel. So the consideration that all the cars have the same size is possible, but inevitably a few special cars will intersperse among them.

Traffic speed is at uniform motion, neglecting the effect of acceleration and deceleration time. Deceleration time in the actual process of vehicles entering the toll station and toll station after the acceleration time is offset each other in the toll plaza shape symmetry. The influence of this term can be ignored.

Cellular represents a physical space which can accommodate standard vehicles and a buffer space. We all know that vehicles usually need to pass the toll plaza at low speed and because of that, it is simple to give the vehicle suitable buffer space. Therefore, our idea is very practical.[3]

The Establishment of Virtual Toll Plaza. In order to represent the merging or diverging lane, we use a simple ladder approximation. The schematic diagram of simple toll plaza is shown in Figure 1. [4]

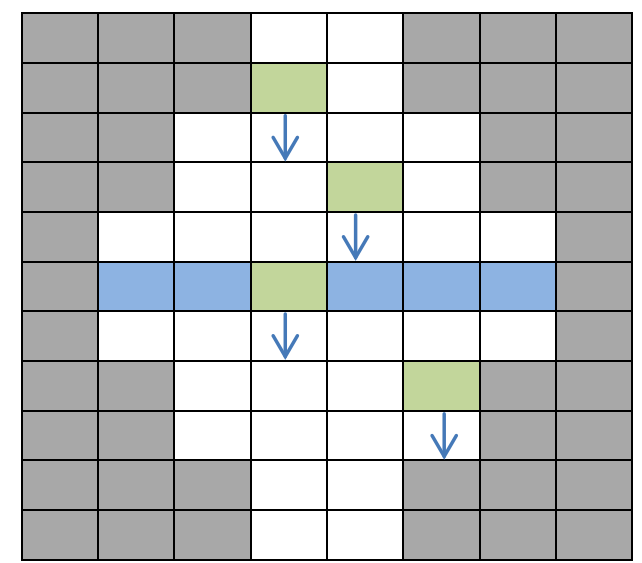

Figure 1 Schematic diagram of simple toll plaza

In Figure 1, the gray grid represents the forbidden cell. The blue grid represents the toll booth. The green grid represents the vehicles, and the arrow indicates the direction of movement.

In order to realize the construction of virtual toll plaza in MATLAB, we create a large matrix to represent the real system. The occupied cell is represented as 1 , while the unoccupied cell is represented as 0 . The forbidden cell is represented as -888 for convenience. The schematic diagram is shown in the following Figure 2.

In this model, the calculation process of each time step is as follows:

(1)From the front of the traffic, we checked each vehicle in turn (according to the flow direction), and each vehicle advances to the next cellular with probability $P$. If the next cellular is not empty, the vehicle will halt and be marked.

(2)The distribution function of incoming traffic can be obtained with, generate a new vehicle on the new border.

(3)Check every vehicle in turn and marked the lane to the left or right with probability $Q$. If the bilateral cellular are occupied, the vehicle will not move.

(4)Calculating the total waiting time.

(5)Clearing away the vehicle on the far boundaries. 


\begin{tabular}{|l|l|l|l|l|l|l|l|}
\hline-888 & -888 & -888 & 0 & 0 & -888 & -888 & -888 \\
\hline-888 & -888 & -888 & 1 & 0 & -888 & -888 & -888 \\
\hline-888 & -888 & 0 & 0 & 0 & 0 & -888 & -888 \\
\hline-888 & -888 & 0 & 0 & 1 & 0 & -888 & -888 \\
\hline-888 & 0 & 0 & 0 & 0 & 0 & 0 & -888 \\
\hline-888 & 0 & 0 & 1 & 0 & 0 & 0 & -888 \\
\hline-888 & 0 & 0 & 0 & 0 & 0 & 0 & -888 \\
\hline-888 & -888 & 0 & 0 & 0 & 1 & -888 & -888 \\
\hline-888 & -888 & 0 & 0 & 0 & 0 & -888 & -888 \\
\hline-888 & -888 & -888 & 0 & 0 & -888 & -888 & -888 \\
\hline-888 & -888 & -888 & 0 & 0 & -888 & -888 & -888 \\
\hline
\end{tabular}

Figure 2The visual representation in the MATLAB of simple toll plaza

The Calculation of Parameters in the Model. Traffic flow. On the basis of previous researches (Quotation),we obtained the distribution function of incoming traffic, which is the distribution of Fourier series. But, in the model, traffic volume of the toll plaza should be an integer and can't exceed the number of available lanes in each time step, so we round for Fourier series and unified units. The Fourier series expression is as follows:[4]

$$
G_{i n}(t)=a_{0}+\sum_{n=1}^{N} a_{n} \cos (n \cdot \omega \cdot t)+\sum_{n=1}^{N} b_{n} \sin (n \cdot \omega \cdot t)
$$

The introduction of the continuous time unit into the time step unit constant factor $\mu$, so rounded and unity of the unit after the inflow function formula is as follows:

$$
G_{i n}^{\prime}(\tau)=\min \left[\operatorname{round}\left(\frac{G_{i n}(t)}{\mu}\right), L\right]
$$

The definition of Boundary Condition. In the model, vehicle can't occupy the boundary and can't cross the boundaries, so we can determine that Boundary Conditions are relative to Dirichlet Boundary. The boundary conditions which are perpendicular to upper boundary of traffic flow, can determine by function of incoming traffic, while the lower boundary can be regarded as ideal absorber, so the vehicle will be clear and be reckon in throughput of toll plaza after it reaches the border.

The calculation of waiting time. Checking the whole matrix on the each time step and recording the number of cellular that has positive value, which is every vehicle's mean waiting time. The expression of it is as follows:

$$
W_{i}=W_{i-1}+1(\operatorname{plaza}(x, y)>0) \forall x, \forall y .
$$

Where: $W_{i}$ - The average waiting time for each vehicle in the i-th time step; plaza-Cell matrix

\section{The Result of the Cellular Automata Model}

We carry out simulated operation for several merging modes. Then, we find the best merging mode is as follow. Merge two adjacent lanes, and then merge them to the main traffic lane. By guiding the flow line on the ground to play the role of guiding traffic, this can not only effectively reduce costs, and will not stop the driver's sight and cause traffic accidents [5]. The merging mode is shown in the following Figure 3. 


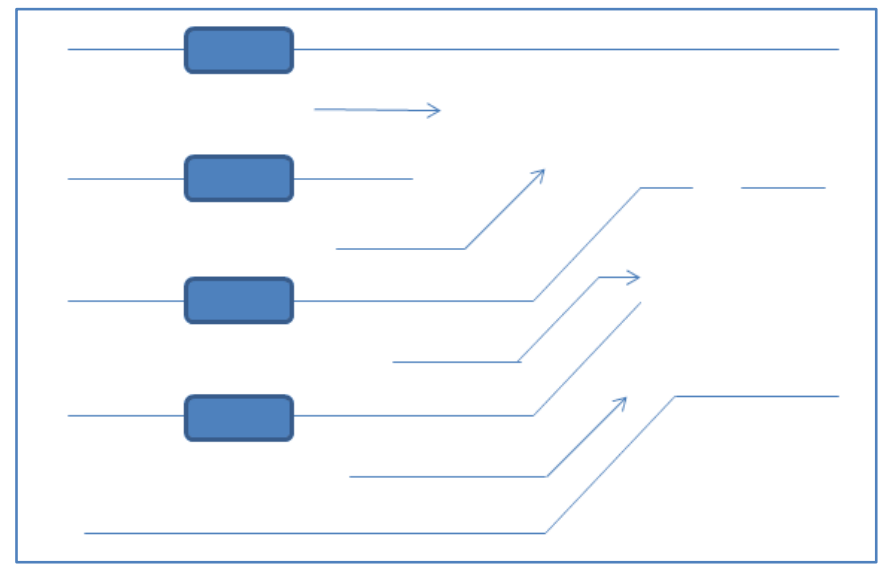

Figure 3 The best merging mode

The boundary condition is added to the previously established cellular automata model, and then we use the program simulation to get the results. The results are shown in Table1:

Table1 The results of the program simulation

\begin{tabular}{|c|c|c|c|}
\hline$t_{0}(\min )$ & $S_{0}$ (vehicle) & $t_{m}(\mathrm{~s})$ & $\varphi$ \\
\hline 4 & 23984 & 10 & 0.014 \\
\hline
\end{tabular}

\section{Conclusion}

Comparisons were made with the results of evaluations not involving merging schemes, so we come to the conclusion: The solution we get, which guides the traffic flow, takes advantage of the area of the toll plaza and increases the area of traffic flow, resulting in improved throughput and reduced average transit time. As the diversion only need to draw the guide line on the ground, so the cost does not increase. Though the increase in throughout resulting in a slight increase in the accident rate, we taking into account the above-mentioned accident rate algorithm error so we can approximate that the accident rate has not risen.

\section{References}

[1] Fu Tian Ren,jiang Rong,Traffic Engineering [M], BeiJing: China Communications Press, 2003

[2]YunTongLiu, HiuXiong, Traffic System Simulation Technology[M], BeiJing: China Communications Press, 2002.

[3] Yuxia Liu, Rongjun Cheng, Xiufang Feng, et al, Cellular Automaton Model Considering Tollbooths with Lane Expansion, Chinese Journal of Theoretical and Applied Mechanics, 2011, 05:844-849.

[4]JessS Boronicoand PhilipHSiegel, Capacity planning for toll road ways incorporating consumer wait time costs, Transportation Research PartA: Policy and Practice, 32(4):297-310,1998.

[5] Xiaoming Shao, Simulation and Application of Energy Consumption for Multi-story Toll Station of High-grade Highway Based on Cellular Automata, Lanzhou Jiaotong University, 2013 